\title{
Description and analysis of 12 years of surveillance for Creutzfeldt-Jakob disease in Denmark, 1997 to 2008
}

S Gubbels (gub@ssi.dk)1,2, S Bacci'1,2, H Laursen³, H Høgenhaven4, S Cowan ${ }^{1}, K_{\text {K Mølbak }}^{1}$, M Christiansen ${ }^{5}$

1. Department of Epidemiology, Statens Serum Institut, Copenhagen, Denmark

2. European Programme for Intervention Epidemiology Training (EPIET), European Centre for Disease Prevention and Control, Stockholm, Sweden

3. Neuropathology Laboratory, Rigshospitalet, Copenhagen, Denmark

4. Department of Clinical Neurophysiology, Rigshospitalet, Copenhagen, Denmark

5. Department of Clinical Biochemistry and Immunology, Statens Serum Institut, Copenhagen, Denmark

Citation style for this article:

Gubbels S, Bacci S, Laursen H, Høgenhaven H, Cowan S, Mølbak K, Christiansen M. Description and analysis of 12 years of surveillance for Creutzfeldt-Jakob disease in Denmark, 1997 to 2008. Euro Surveill. 2012;17(15):pii=20142. Available online: http://www.eurosurveillance.org/ViewArticle.aspx?Articleld=20142

Article submitted on 10 May 2011/ published on 12 April 2012

Prospective surveillance of Creutzfeldt-Jakob disease (CJD) was initiated in Denmark in 1997, following the observation of variant CJD in the United Kingdom. Demographic, clinical and diagnostic information was collected for each patient with clinical suspicion of CJD. Here we describe the methods for surveillance and the observed outcomes between 1 January 1997 and 31 December 2008. A total of 83 patients were classified as sporadic CJD, 47 were definite diagnoses, 34 probable and two possible. This resulted in a mean incidence of 1.26 patients with probable and definite sporadic CJD per million inhabitants. Two sporadic CJD patients were found to have a genetic variant of unknown significance: Thr201Ser and Glu200Asp. One patient was diagnosed with Gerstmann-SträusslerScheinker syndrome. No patients were classified as having variant, iatrogenic or familial CJD. The Danish surveillance system, like those in other countries, has a multidisciplinary approach, which is labour-intensive and time-consuming but ensures the most complete set of information possible. With this approach we think that patients with variant CJD would have been detected had they occurred in Denmark. Certain aspects of CJD surveillance need further discussion at European level and beyond, in order to find a balance between efficiency of the systems and accuracy of surveillance data.

\section{Introduction}

Creutzfeldt-Jakob disease (CJD) is a rare, fatal disorder characterised by rapidly progressive dementia. CJD belongs to the group of transmissible spongiform encephalopathies (TSE) or prion diseases. Four different aetiological subtypes of CJD have been defined: sporadic CJD (sCJD), which is the most common, the familial subtype (fCJD), the iatrogenic subtype (iCJD) and variant CJD (VCJD) [1]. Other related prion diseases are: Gerstmann-Sträussler-Scheinker syndrome (GSS), and fatal familial insomnia (FFI). A definite diagnosis of CJD and distinction between the sporadic and variant type can only be made through post-mortem brain autopsy or biopsy. When no autopsy has been performed, a combination of diagnostic criteria can lead to a probable or possible diagnosis.

In 1996, vCJD was first described in the United Kingdom (UK) [2]. This subtype of the disease was soon linked to bovine spongiform encephalopathy (BSE) [3] and developed into an epidemic, which peaked in the UK in 2000 [4]. Between 1995 and 2008, a total of 205 patients with VCJD were identified worldwide [5].

Mandatory notification of CJD was introduced in several European countries in 1997. Following this, prospective surveillance of TSE was introduced in Danish law the same year [6], making it mandatory for physicians to report patients with clinical suspicion of a TSE to Statens Serum Institut (SSI). In 1998 Denmark became part of the NEUROCJD network, which later merged with the EUROCJD network, a project of the European Union (EU) aimed at coordinating surveillance in Europe, harmonising data collection and providing information on the epidemiological characteristics of CJD.

The purpose of the Danish national surveillance for TSE is to detect and monitor VCJD, in order to be able to take appropriate public health measures. However, due to similarity in clinical features and the rarity of the disease, all subtypes of CJD, and even GSS and $\mathrm{FFI}$, are under surveillance. This article describes the Danish national surveillance system for TSE and provides an epidemiological overview of the outcomes for the years 1997-2008. 


\section{Methods}

During the 12-year period the Department of Epidemiology carried out the surveillance of infectious diseases in Denmark, including surveillance of TSE, on behalf of the National Board of Health. The Department of Epidemiology also regularly informed physicians about the incidence of notified diseases and new developments through the epidemiological bulletin, Epi-News.

\section{Case finding}

If a patient was clinically suspected to have a form of TSE, the Department of Epidemiology of SSI had to be notified. When notified, the Department of Epidemiology sent a supplementary questionnaire to the reporting physician. The questionnaire explored a set of variables on demography, clinical presentation at onset, interpretation of electroencephalography (EEG), magnetic resonance imaging (MRI) and laboratory results. Physicians were also invited to provide additional documentation, in the form of discharge letters, EEG and MRI reports and neuropathological findings following autopsy.

Since 2004 , the $14-3-3$ protein, which reflects nonspecific neuronal damage in the brain when present in cerebrospinal fluid (CSF), has been used for active case-finding [7]. Analysis of CSF was only performed by the Department of Clinical Biochemistry and Immunology at SSI. Samples were identified by a unique patient identifier from the civil registry system [8], which was also used for the mandatory notification. If a patient had a positive 14-3-3 qualitative test and mandatory notification was missing, the Department of Epidemiology contacted the physician. If a TSE diagnosis was considered, the physician was asked to submit the mandatory notification form and questionnaire.

As part of a study on the risk of developing SCJD after surgery, the national hospital discharge and cause of death registers were used to identify patients with probable and definite diagnosis and onset of CJD between 1987 and 2003 [9]. This information was used for active case finding of individuals who were not notified between 1997 and 2003.

\section{Three steps in classification}

Diagnosis of SCJD was retrospectively classified as possible, probable or definite according to the 1998 Rotterdam criteria (Table 1, [10]) and vCJD according to the 2000 UK criteria [11]. For each patient a three-step process was followed for classification. The notifying physician was the first to classify the disease on the questionnaire. Subsequently, the diagnosis was evaluated by a medical epidemiologist at SSI, who may have re-classified on the basis of the available documentation. If necessary, the physician was contacted for further details. An expert panel, consisting of a neuropathologist, a clinical neurophysiologist, a clinical laboratory physician and medical epidemiologists, met yearly to evaluate the notifications and decide on a final classification.

Regular exchange of information between the epidemiologists and the other members of the expert panel during the year ensured an assessment of each notified patient before the annual meeting. However, the classification was subject to change until an agreement was reached by the expert panel during the annual meeting. Patients who were still alive at the time of the annual meeting were followed up until death and discussed in the meeting of the following year for final classification. The surveillance data were based on the year of death of the patient.

TABLE 1

World Health Organization criteria for diagnosis of sporadic Creutzfeldt-Jakob disease

\begin{tabular}{|l|c|l|}
\hline \multicolumn{2}{|l|}{ Criteria } \\
\hline I & & Rapidly progressive dementia \\
\hline II & A & Myoclonus \\
\hline & B & Visual or cerebellar problems \\
\hline & C & Pyramidal or extrapyramidal features \\
\hline III & D & Akinetic mutism \\
\hline Classification & $\begin{array}{l}\text { CJD-typical periodic sharpwave complexes in } \\
\text { electroencephalography }\end{array}$ \\
\hline Definite sCJD & $\begin{array}{l}\text { Requires neuropathological/immunocytochemical } \\
\text { confirmation }\end{array}$ \\
\hline Probable sCJD & $\begin{array}{l}\text { I + two of II + III OR possible sCJD + positive } \\
14-3-3 \text { protein }\end{array}$ \\
\hline Possible sCJD & I + two of II + duration <2 years \\
\hline
\end{tabular}

SCJD: sporadic Creutzfeldt-Jakob disease.

Source: Rotterdam 1998 criteria [10].

\section{Diagnostic methods}

Electroencephalography and magnetic resonance imagin EEG and MRI were performed by the local hospitals for the clinical management of the patient. All EEGs were reviewed by specialists in clinical neurophysiology. A CJD-typical EEG implied that periodic sharpwave complexes (PSWC) had been identified in at least one EEG. For final classification by the expert panel, criteria from the World Health Organization (WHO) were used for EEG reviewing [10]. In the majority of cases the original recordings were reviewed by the expert panel. When this was technically impossible, due to incompatibility of electronic systems, the original reports were reviewed and the local clinical neurophysiologists were contacted when clarifications were needed. Since MRI scans were not part of the classification criteria until January 2010, they were not systematically reviewed by the expert panel, but reports of MRI scans which were available from the patient journals were recorded in the surveillance system for future reference. 
Laboratory techniques

The standard set of tests performed at the clinical laboratory at SSI when CJD was suspected consisted of qualitative 14-3-3 protein detection, supplemented with measurement of neuron-specific enolase (NSE), both of which are markers of neuronal damage, which are released in CSF during the course of different diseases, such as CJD and acute encephalitis. The 14-3-3 protein was detected in CSF using Western blot analysis developed by SSI [7], using antibody raised against the N-terminus of the beta 14-3-3 (sc-629 from Santa Cruz Biotechnology). When the test showed weak bands the analysis was run again. If the band was present the second time the patient was classified as 14-3-3 positive, irrespective of the size of the band. NSE levels were determined using time-resolved amplified cryptate emission (TRACE) technology [7]. NSE levels were considered elevated for CJD diagnosis if they were above $35 \mathrm{ng} / \mathrm{ml}$. In case of a discrepancy between the results of the $14-3-3$ and NSE analyses the 14-3-3 result was the decisive marker.

In addition, physicians had the option to request sequence analysis of the prion protein gene (PRNP) on genomic DNA isolated from blood. The PRNP gene was sequenced to establish the genotype at known polymorphic loci, i.e. codon 129 and 219, which have been ascribed a role as disease modifiers [12], and to identify putative disease-causing mutations. Specific genetic variants are relatively prevalent in certain populations and the occurrence of such mutations was established as part of the surveillance in cases where DNA material from whole blood was available. Genetic analysis was performed according to previously described standard procedures [13].

\section{Autopsy}

Autopsy, necessary to confirm or exclude the diagnosis, could be performed when family consent was given. Autopsies were performed at local hospitals or at the National Reference Centre for Creutzfeldt-Jakob Disease and other Spongiform Encephalopathies, Neuropathology Laboratory, Rigshospitalet, Copenhagen. In most cases the neuropathological investigation was performed at the National Reference Centre. The brains were fixed in $10 \%$ formalin for six to eight weeks before cutting. Paraffin blocs from all cerebral lobes, striatum, thalamus, hippocampus, brain stem, pons, cerebellum and medulla were scrutinised using hematoxylin and eosin, luxol fast blue, Periodic acid-Schiff and other conventional stains. Immunohistochemistry included staining for betaamyloid, tau, p-tau, alfa-synuclein, ubiquitin, GFAp, CD68, NF, and for prion proteins (PrP) with $3 \mathrm{~F}_{4}$ and $\mathrm{KG}_{9}$ antibodies. Also, paraffin-embedded tissue blot was applied in some cases.
Data analyses

Surveillance data of CJD patients collected from 1 May 1997 to 31 December 2008 were used for these analyses. All notified patients classified as possible, probable and definite were included. Results for 14-3-3 protein and NSE levels were available from 1 January 1998 to 31 December 2008, and genetic analysis of PRNP from 1 January 2001 to 31 December 2008.

Incidence of probable and definite diagnoses was calculated using the population size of Denmark on 1 January of each year available from the Danish Office of National Statistics [14]. Only probable and definite diagnoses of CJD were included in the calculation of incidence, since that is the measure that was reported in the EUROCJD surveillance and allowed the comparison of our data with other countries. The incidence could also be compared to countries reporting annual mortality since CJD has a short duration of illness and always leads to death. Poisson regression with an interaction term was applied to compare the agespecific incidence between men and women. One-way analysis of variance (ANOVA) was used to analyse the duration of illness by age group and Mann-Whitney test to compare the duration of illness among men and women. The level of significance was set at 0.05 .

\section{Results}

Of the patients notified between 1 May 1997 and 31 December 2008, 83 were classified as SCJD and one as GSS. No patients were classified as variant, iatrogenic or familial CJD or FFI. Clinical symptoms at onset and demographic characteristics were described for all 83 SCJD patients, EEG findings for 81 patients and MRI findings for 63 patients. Information related to CSF investigation was available for 52 patients concerning 14-3-3 protein and for 51 concerning the NSE. Polymorphism and genetic analyses were available for 43 and 39 patients, respectively.

\section{FIGURE 1}

Patients with sporadic Creutzfeldt-Jakob disease by year of death and final classification, Denmark, 1997-2008 $(\mathrm{n}=83)$

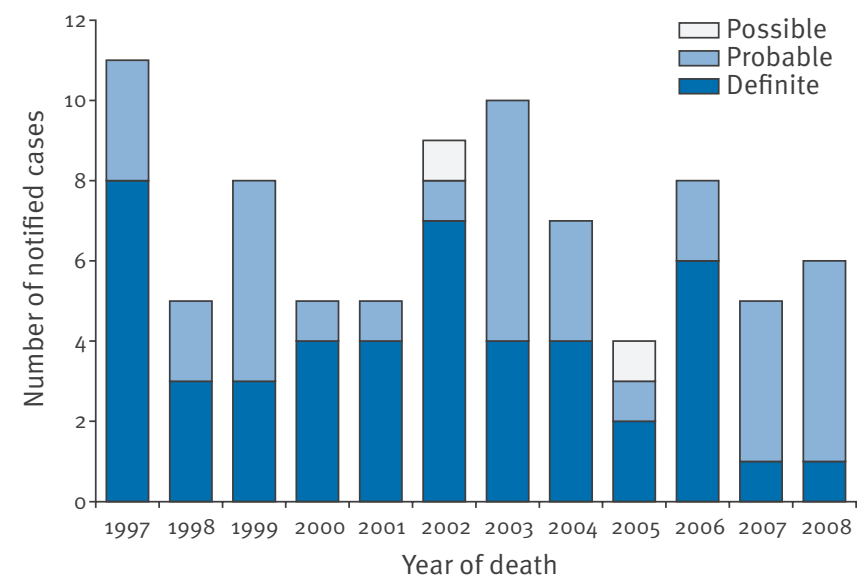




\section{Demographic description}

Among the $83 \mathrm{sCJD}$ patients 47 (57\%) were classified as definite, $34(41 \%)$ as probable and two $(2 \%)$ as possible. Forty-four SCJD patients were male (53\%) and 39 female (47\%). All patients were residents in Denmark. Figure 1 shows the number of patients by year of death and by final classification. The average number of possible, probable and definite SCJD patients per year was seven. The proportion of definite diagnoses in the total number of SCJD patients (definite, probable, possible) during the first 10 years of the surveillance varied between $38 \%$ in 1999 and $80 \%$ in 2000 and 2001, after which it declined to $20 \%$ in 2007 and $17 \%$ in 2008 .

With a mean population size of 5.4 million inhabitants during the 12 year period, the mean incidence of probable and definite SCJD patients in Denmark over this period was 1.26 per million ( $95 \%$ confidence interval (CI): 1.01-1.56), and varied between 2.09 per million in 1997 and 0.55 per million in 2005. The median age at onset of disease for patients with probable and definite diagnosis was 66 years (range: $40-88$ years). Figure 2 shows the incidence of the 81 patients with definite and probable diagnosis stratified by sex and age at onset. Incidence increased with age until it peaked at 70-79 years and dramatically dropped for persons older than 80 years. No women older than 80 years were reported with CJD. Although there was a higher incidence of SCJD among men than women in the age groups 70-79 years and $>80$ years, the age-specific incidence was not significantly different between men and women $(p=0.187)$.

The median duration of illness from onset until death for the 81 probable and definite SCJD patients was 3.8 months (range: 1.2-21.8 months). Older age was associated with shorter duration of illness (ANOVA, $p=0.002$ ). There was no association between duration of disease and sex (Mann-Whitney $p=0.12$ ).

\section{FIGURE 2}

Incidence of definite and probable sporadic CreutzfeldtJakob disease, by sex and age at onset, Denmark,

$1997-2008 \quad(\mathrm{n}=81)$

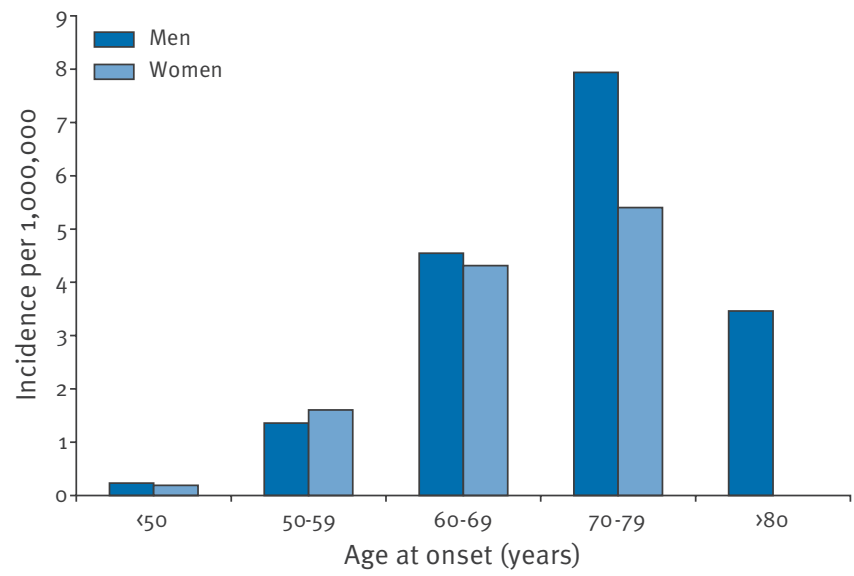

Diagnostic findings

Table 2 describes the different diagnostic findings among the definite, probable and possible SCJD patients. A majority of 67 patients ( $81 \%$ ) presented with rapidly progressive dementia at onset, without other cognitive or physical symptoms present alone during the first two weeks of illness. Of the 14 patients with other presentations, nine were confirmed as SCJD with autopsy. For three patients with a probable diagnosis, the primary clinical presentation was classified as Heidenhain's syndrome, a visual impairment which leads to cortical blindness. No patients were seen with a pure psychiatric onset.

Of 82 patients for whom an EEG report was available 58 were reported to have an EEG typical for SCJD. Among the 46 patients with a definite diagnosis 31 had an EEG typical for SCJD; among the 34 with a probable diagnosis the proportion of patients with a typical EEG was higher with 27 patients.

For 63 patients an MRI report was also available and 43 of these patients showed abnormalities. Atrophy and unspecific abnormalities were most commonly reported, in 26 and 27 patients, respectively. Hyperintensity in the caudatus and putamen was less common with 17 patients, and hyperintensity in the thalamus was only reported in two patients.

For 52 patients $14-3-3$ protein was tested and 41 had a positive result. Of the 20 definite cases seven had a negative 14-3-3 result. NSE levels were available for 51 patients; of these 37 had elevated levels above $35 \mathrm{ng} / \mathrm{ml}$.

Polymorphisms in codons 129 and 219 were examined for 43 patients. The distribution of amino acids on codon 129 was methionine (Met) homozygocity for 24 patients, valine (Val) homozygocity for 11 patients and Met/Val heterozygocity for eight patients. Codon 219 showed homozygocity for glutamic acid (Glu) in all 43 patients. Table 3 shows the EEG results for 43 patients for whom the polymorphisms were tested. A majority of 21 of 24 patients with Met/Met at codon 129 had a typical EEG (Table 3). This proportion was smaller among patients with Met/Val (four of eight). Only one of 10 patients with Val/Val had a typical EEG.

In addition, 39 patients were tested for genetic variants (Table 2). No octarepeat variants were found among these patients. In two CJD patients we found a PRNP mutation. The first patient had a definite SCJD diagnosis and showed a new mutation in codon 201 from threonine (Thr) to serine (Ser), resulting in homozygosity for Ser. On codon 129 this patient had a Met/Met polymorphism. Microsatellites were tested on both sides of the gene, confirming that no deletion was present. The second patient had a probable SCJD diagnosis and showed a mutation from Glu to aspartic acid (Asp) in codon 200. This patient was heterozygous for Met/ $\mathrm{Val}$ at codon 129. The patients were referred to local 
TABLE 2

Diagnostic characteristics of patients with definite, probable and possible sporadic Creutzfeldt-Jakob disease, Denmark, 1997-2008 $(\mathrm{n}=83)$

\begin{tabular}{|c|c|c|c|c|c|}
\hline & \multirow{2}{*}{$\begin{array}{c}\text { Definite } \\
n\end{array}$} & \multirow{2}{*}{$\begin{array}{c}\text { Probable } \\
n\end{array}$} & \multirow{2}{*}{$\begin{array}{c}\text { Possible } \\
n\end{array}$} & \multicolumn{2}{|c|}{ Total } \\
\hline & & & & $\mathrm{n}$ & $\%$ \\
\hline Clinical presentation at onset ${ }^{\mathrm{a}}$ & 47 & 34 & 2 & 83 & \\
\hline (a) Rapidly progressive dementia & 36 & 29 & 2 & 67 & $81 \%$ \\
\hline (b) Heidenhain's syndrome & 0 & 3 & 0 & 3 & $4 \%$ \\
\hline (c) Pure psychiatric onset & 0 & 0 & 0 & 0 & $0 \%$ \\
\hline (d) Slowly progressive dementia & 1 & 0 & 0 & 1 & $1 \%$ \\
\hline (e) Pure cerebellar onset & 4 & 1 & 0 & 5 & $6 \%$ \\
\hline (f) Extrapyramidal onset & 2 & 1 & 0 & 3 & $4 \%$ \\
\hline (g) Stroke-like onset & 1 & 0 & 0 & 1 & $1 \%$ \\
\hline (h) Sensory symptoms at onset & 1 & 0 & 0 & 1 & $1 \%$ \\
\hline (i) Not possible to categorise & 2 & 0 & 0 & 2 & $2 \%$ \\
\hline $\mathrm{EEG}^{\mathrm{b}}$ & 46 & 34 & 2 & 82 & \\
\hline EEG typical for sCJD & 31 & 27 & 0 & 58 & $71 \%$ \\
\hline$M R I^{b}$ & 34 & 27 & 2 & 63 & \\
\hline Abnormal MRI & 22 & 19 & 2 & 43 & $68 \%$ \\
\hline Hyperintense caudatus/putamen & 8 & 7 & 2 & 17 & $27 \%$ \\
\hline Hyperintense thalamus & 0 & 2 & 0 & 2 & $3 \%$ \\
\hline Atrophy & 14 & 11 & 1 & 26 & $41 \%$ \\
\hline Unspecific abnormalities & 15 & 10 & 2 & 27 & $43 \%$ \\
\hline 14-3-3 protein in cerebro-spinal fluid ${ }^{b}$ & 27 & 24 & 1 & 52 & \\
\hline Positive for $14-3-3$ & 20 & 21 & 0 & 41 & $79 \%$ \\
\hline Neuron-specific enolase in cerebro-spinal fluid ${ }^{b}$ & 26 & 24 & 1 & 51 & \\
\hline Neuron-specific enolase elevated $(\geq 35 \mathrm{ng} / \mathrm{ml})$ & 18 & 19 & 0 & 37 & $73 \%$ \\
\hline Polymorphisms ${ }^{b}$ & 22 & 20 & 1 & 43 & \\
\hline Codon 129: Met/Met & 12 & 12 & 0 & 24 & $58 \%$ \\
\hline Codon 129: Met/Val & 2 & 5 & 1 & 8 & $19 \%$ \\
\hline Codon 129: Val/Val & 8 & 3 & 0 & 11 & $26 \%$ \\
\hline Codon 219: Glu/Glu & 22 & 20 & 1 & 43 & $100 \%$ \\
\hline Genetic variants $^{b}$ & 19 & 19 & 1 & 39 & \\
\hline Octarepeat variants & 0 & 0 & 0 & 0 & $0 \%$ \\
\hline PRNP mutation & 1 & 1 & 0 & 2 & $5 \%$ \\
\hline
\end{tabular}

EEG: electroencephalography; MRI: magnetic resonance imaging; PRNP: prion protein gene; sCJD: sporadic Creutzfeldt-Jakob disease.

a Description of the categories of symptoms at onset [10]:

(a) Encephalopathic illness with dementia and diverse other neurological features, progressing rapidly over weeks to a few months, with no individual cognitive or physical deficit being present alone for more than two weeks.

(b) Impairment of visual acuity and/or field, progressing to cortical blindness, without other significant clinical deficit for the first two weeks of illness.

(c) Psychiatric symptoms, without the presence of other features for at least four weeks.

(d) Dementia developing over months to years, without any other significant neurological features for the first six months.

(e) Progressive cerebellar syndrome without other significant features, for at least two weeks.

(f) An extrapyramidal syndrome involving Parkinsonian features with or without chorea, athetosis or dystonia, but without other significant features for at least two weeks.

(g) Onset is abrupt enough for a diagnosis of stroke in the initial stages.

(h) Somato-sensory symptoms alone for at least two weeks.

(i) These patients had a complicated medical history, which made it impossible to categorise.

b These diagnostic characteristics were not available for all 83 patients. 


\section{TABLE 3}

Electroencephalography results and polymorphisms in codon 129 for patients with definite, probable and possible sporadic Creutzfeldt-Jakob disease $(n=42)$

\begin{tabular}{|l|c|c|c|c|c|c|}
\hline \multirow{2}{*}{} & \multicolumn{2}{|c}{ Met/Met } & \multicolumn{2}{c|}{ Met/Val } & \multicolumn{2}{c|}{ Val/Val } \\
\cline { 2 - 7 } & $\begin{array}{c}\text { Typical } \\
\text { EEG }\end{array}$ & Total & $\begin{array}{c}\text { Typical } \\
\text { EEG }\end{array}$ & Total & $\begin{array}{c}\text { Typical } \\
\text { EEG }\end{array}$ & Total \\
\hline Definite & 9 & 12 & 0 & $\mathbf{2}$ & 1 & 7 \\
\hline Probable & 12 & 12 & 3 & 5 & 0 & 3 \\
\hline Possible & 0 & 0 & 1 & 1 & 0 & 0 \\
\hline Total & $\mathbf{2 1}$ & $\mathbf{2 4}$ & $\mathbf{4}$ & $\mathbf{8}$ & $\mathbf{1}$ & $\mathbf{1 0}$ \\
\hline
\end{tabular}

clinical genetics departments for clinical assessment and to explore the family history.

One patient was found to have GSS. At the age of 58 he developed what was considered a myelopathy. Over the following years the patient further developed gait disturbances, ataxia, dysarthria, double vision and difficulties swallowing. For a long time a form of spinocerebellar ataxia was suspected, but all genetic analyses for that as well as EEGs and computed tomography (CT) scans were normal. Eventually, the patient developed dementia. At autopsy characteristic spongiforme encephalopathy with proteinase K-resistant PrPpositive plaques was diagnosed and a $\mathrm{P} 102 \mathrm{~L}$ mutation was demonstrated in the PRNP gene.

\section{Sources of notification}

Between 1997 and 2008, 70 of the 83 patients with an SCJD diagnosis were reported through the standard mandatory notification system (Figure 3). One patient was notified by the neuropathologist of the expert panel. The study of the national hospital discharge and

\section{FIGURE 3}

Initial source of surveillance referral of patients with possible, probable and definite sporadic Creutzfeldt-Jakob disease, Denmark, 1997-2008 ( $\mathrm{n}=83$ ), by year of death, 1997-2008, Denmark $(\mathrm{n}=83)$

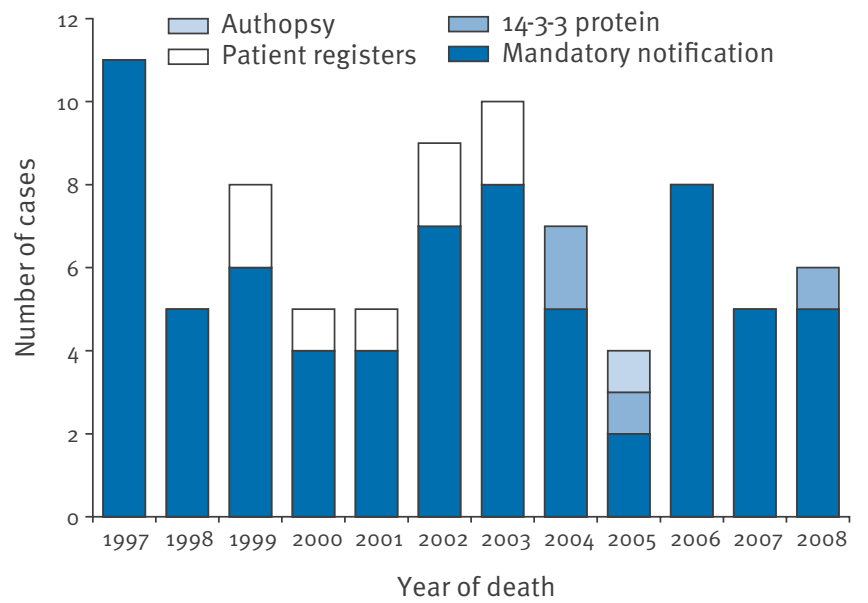

cause-of-death registers identified eight additional SCJD patients who died in the period between 1997 and 2003. Active case-finding with the 14-3-3 test, which started in 2004, identified 26 patients with a positive result (data not shown). After careful evaluation of each of these patients, only four were classified as having SCJD (Figure 3).

The time between onset of illness and notification differed between the sources of notification. For the mandatory notification system the median time was four months (range $0.8-32.9$ months), whereas the median time between onset and notification by a physician after active case finding with a positive 14-3-3 test was 8.8 months (range 3.5-25.8 months). The one patient who was notified by the neuropathologist had onset of symptoms 14.1 months before the notification.

\section{Discussion}

The Danish national surveillance system for CJD integrates the expertise of different professionals and has a multidisciplinary approach. In this paper we describe 12 years of Danish CJD surveillance. We provide a demographic, diagnostic and clinical overview of notified patients and discuss the accuracy of the surveillance methods used.

Our assessment is subject to some limitations. The decision to ask for certain diagnostic tests and to notify a patient depends on the clinical presentation of the patient and the management of individual physicians. Therefore, the availability of diagnostic tests, especially the genetic analyses, might have been biased. Nevertheless, the Danish surveillance system was able to collect a rather complete set of information for each patient. The completeness of the dataset varied between $75 \%$ and $100 \%$ for variables related to demography, clinical symptoms, EEG and MRI.

Consistent with the fact that $\mathrm{SCJD}$ is the most common form of TSE, 83 of 84 notified patients in the Danish surveillance were diagnosed with SCJD and only one with GSS. The mean incidence of 1.26 definite and probable cases per million people per year is consistent with the observed overall annual mortality of $\mathbf{1 . 3 9}$ per million for SCJD in Europe, Australia and Canada [15]. As CJD has a short duration of illness always leading to death, the incidence and annual mortality can be compared. The incidence was highest among persons between 70 and 79 years of age and dropped dramatically among persons older than 80 years. This pattern is also described by other European countries as well as Canada, Australia, Taiwan and Japan [15-19]. Although the drop in SCJD incidence among women in the oldest age group was more pronounced in the Danish surveillance than described by other countries, the difference between women and men of that age was not statistically significant. This dramatic drop after the age of 80 could reflect the real disease epidemiology. Although clinical and neuro-biochemical features are similar among the age groups [20], another explanation could 
be that the disease was misclassified in the older age groups, for example as Alzheimer's disease, for which the incidence steadily rises with age [21].

Rapidly progressive dementia is typically the first presentation in patients with CJD [22] and was also the most commonly reported as presentation at onset in our surveillance. The finding that $4 \%$ of the patients had Heidenhain's syndrome at onset is consistent with observations from a large group of British patients [23]. The typical PSWC in the EEG occur in about two thirds of patients with SCJD and $\mathrm{ICJD}$, in $10 \%$ of patients with $\mathrm{fCJD}$, but not in patients with VCJD [24]. The overall finding that 58 patients of 82 had a typical EEG is comparable to those data. Wieser et al. described that PSWC occur most often in patients with Met homozygosity at codon 129 of the PRNP gene and only occasionally in patients with Met/Val heterozygosity and Val homozygoty [24]. A similar distribution was seen in our surveillance data.

Zerr et al. found that the highest diagnostic accuracy of the MRI scan was obtained when either a combination of at least two cerebral cortical regions (temporal, occipital or parietal) show an increased signal or when a high signal increase is observed in both the caudate nucleus and the putamen [25]. These findings led to an adjustment in the classification criteria used within the EUROCJD network as of 1 January 2010, adding a high signal in caudate nucleus and/or putamen to the criteria on the same level as PSWC in the EEG. Interestingly, among the Danish patients only 17 of 63 patients showed a high signal in caudate nucleus and/ or putamen. This may be due to the fact that reports were not systematically reviewed and shows the need to implement systematic review of MRI scans now that MRI scans have been added as a diagnostic criterion for CJD.

Polymorphisms at codon 129 have a strong modifying effect on disease susceptibility and phenotype [26]. Individuals homozygous for Met or Val at codon 129 are susceptible to developing SCJD [12]. The proportion of Danish SCJD patients homozygous for Met was indeed higher than the $35 \%$ in the healthy Danish population [27]. Lysine (Lys) at codon 219 has been suggested to be a protective factor against SCJD [28], and has only been shown in Asian and Pacific populations [29]. The finding that all SCJD patients who had a genetic analysis in our surveillance system were homozygous for Glu in codon 219, was therefore not surprising.

The homozygous Thr/Ser mutation in codon 201 was associated with Met/Met polymorphism in codon 129 and a deletion was excluded on microsatellite analysis. The Glu/Asp mutation in codon 200 was associated with Met/Val on codon 129. The Glu/Asp mutation occurred in the same codon as the mutation Glu/ Lys which has been found in more than $70 \%$ of $\mathrm{fCJD}$ patients [30]. However, neither the Glu/Asp mutation nor the Thr/Ser mutation have been described before as associated with $\mathrm{fCJD}$. It is therefore likely that these were novel mutations rather than mutations indicating $\mathrm{fCJD}$, although the family history of these patients was not available to further assess this statement. The fact that the Thr/Ser mutation was homozygous suggest even more strongly that it was a novel one, as the chance of inheriting the same unknown mutation from both parents is extremely low. The clinical significance of novel mutations is difficult to establish as insignificant missense mutations do tend to occur in the PRNP gene. Considering this, we classified both patients as sCJD patients, with the addition that they had a genetic variant of unknown significance.

The patient with GSS had the classical morphology and proteinase K-resistant PrP-positive plaques previously described [1]. Also, the $\mathrm{P}_{102} \mathrm{~L}$ mutation is the same as the one identified in the family from Vienna, which was first described to have GSS [31]. The Danish case is considered to represent the 34th known family in the world with the disease.

Surveillance systems for TSE have been described for Germany, France and Belgium $[16,17,20]$. In France the surveillance has identified $25 \mathrm{vCJD}$ patients to date, while Germany and Belgium have not found any vCJD patients [5]. In Germany, suspected CJD patients were identified by referral from the treating physician, or after discussion of the 14-3-3 test result with a surveillance neurologist [16]. French patients suspected to have CJD were either notified by the physician or, more often, by a laboratory following a request for a 14-3-3 test [17]. In Belgium, all patients in the seven collaborating university hospitals clinically suspected of having probable CJD were reported. Patients with a clinical suspicion from other hospitals were identified through the 14-3-3 test [20]. Like the Danish system, the systems in Germany, France and Belgium are based on a multidisciplinary approach which operates on a central level and involves detailed traceback of patient files, laboratory results and diagnostic investigations such as EEG, MRI and neuropathology. These systems also use the 14-3-3 test, although they apply it in different ways. In Denmark, active case-finding with 14-3-3 has identified four additional patients over a five-year period, and 22 false positives. With such a low yield it is questionable whether the use of $14-3-3$ test for active case finding is effective. Even more so, because the 14-3-3 test is often false negative in vCJD and the use of 14-3-3 protein therefore does not improve the sensitivity of the surveillance system for vCJD [32]. An advantage of using the 14-3-3 test for active case finding is that it reminds physicians of the mandatory notification and maintains awareness. If the 14-3-3 test was removed from the surveillance system as a source of notification, it would be of utmost importance to regularly remind physicians of the need to notify suspected cases, as the system would then fully rely on the mandatory notification. The multidisciplinary approach with traceback of files and additional case finding is a time-consuming and labour-intensive 
method. It may, however, be the only way to identify as many CJD cases as possible, and it minimises variation in how the different variables in a complex classification process are interpreted. Algorithms and sensitivity analyses have been encouraged in order to enhance clinical diagnosis [7]. By supporting the physician's diagnosis, those algorithms could contribute to a more efficient surveillance.

With monitoring of vCJD as the main aim of the surveillance, it is important to examine whether not finding any VCJD in Denmark is a true indication that there really were no cases. Denmark is an agricultural country and only a small proportion of cattle and beef are imported. Since the surveillance of BSE in cattle started in 1990, 15 animals with BSE have been identified in Denmark, and an additional three in exported Danish cattle [33]. These are very low numbers compared to 184,600 cattle with BSE in the UK and 1,006 in France between 1988 and 2008 [34]. Respectively, 164 and 23 patients with vCJD were notified in these countries between 1995 and 2008 [5]. With the low number of cattle diagnosed with BSE in Denmark, despite thorough testing, it is unlikely that human cases of vCJD exist. In addition, it is unlikely that patients with vCJD have been misclassified as SCJD in our system, considering that the patients we report here were older than vCJD patients described in France and the UK (mean age 37 and 30 years, respectively) [35]. None of our patients was younger than 40 years at disease onset. Moreover, none of the Danish patients were reported to have psychiatric symptoms at onset, which is the most typical presentation of vCJD [11].

A median delay of four months was observed between onset of disease and notification. This could be caused by the complicated diagnostic process, but is also likely to involve a delay between clinical suspicion of diagnosis and notification. Active case finding through the 14-3-3 test did not shorten this delay.

The number of autopsies performed on patients with a probable and possible diagnosis declined, leading to a lower number of definite diagnoses and a larger uncertainty in the surveillance. An American study showed that reluctance of the family to give consent was one of the most important barriers to performing an autopsy [36]. This does not, however, explain why the number of autopsies decreased over those 12 years. Physicians might have been less likely to arrange for an autopsy in the more recent years, compared with the time shortly after the VCJD epidemic in the UK. The decline in autopsies increases the need for accurate clinical and laboratory data to assess the number of possible and probable diagnoses.

In conclusion, the Danish CJD surveillance registered an incidence of 1.26 probable and definite SCJD cases per million between 1997 and 2008. No patients with vCJD were found. The observed delay of four months between onset and notification could be of concern for public health measures should a patient with vCJD be detected. Awareness among physicians needs constant attention as it is important for timely notification as well as for the number of autopsies performed. In addition, it is important to further assess the cost effectiveness of the surveillance, with a view to the labour-intensive methods and the use of 14-3-3 protein for active case finding. We therefore recommend evaluating TSE surveillance in a broader context and generating discussion on a European level and beyond.

\section{Acknowledgments}

We wish to thank all physicians who notified patients and provided us with the necessary information. We also thank Linda Roth and Gerhard Falkenhorst (Department of Epidemiology, SSI, Copenhagen, Denmark) for their involvement in carrying out the surveillance, Cathrine Jespersgaard and Michael Pfeiffer (Department of Clinical Biochemistry and Immunology, SSI, Copenhagen, Denmark) for the laboratory testing and Oktawia Wojcík (European Programme for Intervention Epidemiology Training (EPIET), European Centre for Disease Prevention and Control, Stockholm, Sweden and Department of Epidemiology, SSI, Copenhagen, Denmark) and Marion Muehlen (European Programme for Intervention Epidemiology Training (EPIET), European Centre for Disease Prevention and Control, Stockholm, Sweden) for their input to the manuscript.

\section{Funding}

This work was part of routine surveillance and was therefore not supported with additional funding.

\section{References}

1. Tyler KL. Prions and Prion Diseases of the Central Nervous System (Transmissible Neurodegenerative Diseases) - Chapte 175. In: Mandell GL, Bennett JE, Dolan R, editors. Mandell, Douglas and Bennett's principles and practice of infectious diseases 2. New York: Churchill Livingstone; 2005. p. 2219-35.

2. Will RG, Ironside JW, Zeidler M, Cousens SN, Estibeiro K, Alperovitch A, et al. A new variant of Creutzfeldt-Jakob disease in the UK. Lancet. 1996;347(9006):921-5.

3. Bruce ME, Will RG, Ironside JW, McConnell I, Drummond $D$, Suttie A, et al. Transmissions to mice indicate that "new variant" CJD is caused by the BSE agent. Nature. 1997;389(6650):498-501.

4. National Creutzfeldt-Jakob Disease Surveillance Unit (NCJDSU). CID Statistics. Edinburgh: NCJDRSU. [Accessed 10 Aug 2010]. Available from: http://www.cjd.ed.ac.uk/figures.htm

5. European Creutzfeldt Jakob Disease Surveillance Network. EUROCJD Surveillance Data. Edinburgh: EUROCJD; 2011. Available from: http://www.eurocjd.ed.ac.uk/surveillance $\% 20$ data\%204.htm

6. Strandberg Pedersen N. Mad cows and Creutzfeldt-Jakob Disease. EPI-NEWS. 1997:10. Copenhagen: Statens Serum Institut; 1997.

7. Bahl JMC, Heegaard NHH, Falkenhorst G, Laursen H, Høgenhaven H, Mølbak K, et al. The diagnostic efficiency of biomarkers in sporadic Creutzfeldt-Jakob disease compared to Alzheimer's disease. Neurobiol. Aging. 2009;30(11):1834-41.

8. Pedersen CB, Gøtzsche H, Møller JO, Mortensen PB. The Danish Civil Registration System. A cohort of eight million persons. Dan Med Bull. 2006;53(4):441-9. 
9. Mahillo-Fernandez I, de Pedro-Cuesta J, Bleda MJ, Cruz M, Mølbak K, Laursen H, et al. Surgery and risk of sporadic Creutzfeldt-Jakob disease in Denmark and Sweden registry-based case-control studies. Neuroepidemiology. 2008;31(4):229-40.

10. World Health Organization. Human transmissible spongiform encephalopathies. Wkly. Epidemiol. Rec. 1998;73(47):361-5

11. Will RG, Zeidler M, Stewart GE, Macleod MA, Ironside JW, Cousens SN, et al. Diagnosis of new variant Creutzfeldt-Jakob disease. Ann. Neurol. 2000;47(5):575-82.

12. Mead S. Prion disease genetics. Eur. J. Hum. Genet. 2006;14(3):273-81

13. Windl O, Giese A, Schulz-Schaeffer W, Zerr I, Skworc K, Arendt S, et al. Molecular genetics of human prion diseases in Germany. Hum. Genet. 1999;105(3):244-52.

14. Population and elections. Copenhagen: Danmarks Statistik; 2011. [Accessed 10 Aug 2010]; Available from: http://www. statbank.dk/statbank5a/selecttable/omradeo.asp?SubjectCod $\mathrm{e}=02 \&$ PLanguage $=1 \&$ ShowNews $=$ OFF

15. Ladogana A, Puopolo M, Croes EA, Budka H, Jarius C, Collins $\mathrm{S}$, et al. Mortality from Creutzfeldt-Jakob disease and related disorders in Europe, Australia, and Canada. Neurology. 2005;64(9):1586-91.

16. Heinemann U, Krasnianski A, Meissner B, Varges D, Kallenberg K, Schulz-Schaeffer WJ, et al. Creutzfeldt-Jakob disease in Germany: a prospective 12-year surveillance. Brain. 2007;130(Pt 5):1350-9.

17. Brandel J-P, Salomon D, Capek I, Vaillant V, Alpérovitch A. [Epidemiological surveillance of Creutzfeldt-Jakob in France]. Rev. Neurol. (Paris). 2009;165(8-9):684-93. French.

18. Lu C-J, Sun Y, Chen S-S. Incidence of Creutzfeldt-Jakob disease in Taiwan: a prospective 10-year surveillance. Eur. J. Epidemiol. 2010;25(5):341-7.

19. Nakamura Y, Yanagawa H, Hoshi K, Yoshino H, Urata J, Sato T. Incidence rate of Creutzfeldt-Jakob disease in Japan. Int J Epidemiol. 1999;28(1):130-4

20. Van Everbroeck B, Michotte A, Sciot R, Godfraind C, Deprez M, Quoilin S, et al. Increased incidence of sporadic CreutzfeldtJakob disease in the age groups between 70 and 90 years in Belgium. Eur. J. Epidemiol. 2006;21(6):443-7.

21. Ott A, Breteler MM, van Harskamp F, Stijnen T, Hofman A. Incidence and risk of dementia. The Rotterdam Study. Am. J. Epidemiol. 1998;147(6):574-80.

22. Tyler K. Prions and prion diseases of the central nervous system (transmissible neurodegen-erative diseases) In: Mandell GL, Bennett JE, Dolin R, editors. Mandell, Douglas and Bennett's principles and practice of infectious diseases. 6th ed. New York [u.a.]: Churchill Livingstone; 2005.

23. Cooper SA, Murray KL, Heath CA, Will RG, Knight RSG. Isolated visual symptoms at onset in sporadic Creutzfeldt-Jakob disease: the clinical phenotype of the "Heidenhain variant." $\mathrm{Br}$ J Ophthalmol. 2005;89(10):1341-2.

24. Wieser HG, Schindler K, Zumsteg D. EEG in Creutzfeldt-Jakob disease. Clin Neurophysiol. 2006;117(5):935-51.

25. Zerr I, Kallenberg K, Summers DM, Romero C, Taratuto A, Heinemann U, et al. Updated clinical diagnostic criteria for sporadic Creutzfeldt-Jakob disease. Brain. 2009;132(Pt 10):2659-68.

26. Palmer MS, Dryden AJ, Hughes JT, Collinge J. Homozygous prion protein genotype pre-disposes to sporadic CreutzfeldtJakob disease. Nature. 1991;352(6333):340-2.

27. Dyrbye H, Broholm H, Dziegiel MH, Laursen H. The M129V polymorphism of codon 129 in the prion gene $(P R N P)$ in the Danish population. Eur. J. Epidemiol. 2008;23(1):23-7.

28. Shibuya S, Higuchi J, Shin RW, Tateishi J, Kitamoto T. Codon 219 Lys allele of $P R N P$ is not found in sporadic CreutzfeldtJakob disease. Ann. Neurol. 1998;43(6):826-8.

29. Soldevila M, Calafell F, Andrés AM, Yagüe J, Helgason A, Stefánsson K, et al. Prion susceptibility and protective alleles exhibit marked geographic differences. Hum. Mutat. 2003;22(1):104-5.

30. Lee HS, Sambuughin N, Cervenakova L, Chapman J, Pocchiari $M$, Litvak S, et al. Ancestral origins and worldwide distribution of the PRNP $200 \mathrm{~K}$ mutation causing familial Creutzfeldt-Jakob disease. Am. J. Hum. Genet. 1999;64(4):1063-70.

31. Hainfellner JA, Brantner-Inthaler S, Cervenáková L, Brown P, Kitamoto T, Tateishi J, et al. The original Gerstmann-SträusslerScheinker family of Austria: divergent clinicopathological phenotypes but constant PrP genotype. Brain Pathol. 1995;5(3):201-11.

32. Green AJE, Ramljak S, Müller WEG, Knight RSG, Schröder HC. 14-3-3 in the cerebrospinal fluid of patients with variant and sporadic Creutzfeldt-Jakob disease measured using capture assay able to detect low levels of 14-3-3 protein. Neurosci. Lett. 2002 10;324(1):57-60.

33. Rugbjerg H. Creutzfeldt-Jakobs Disease (CJD). Bovine spongiforme encephalopaty (BSE). EPI-NEWS. 2010:16. Copenhagen: Statens Serum Institut; 2010. [Accessed 16 Aug 2010]. Available from: http://www.ssi.dk/English/News/EPINEWS $/ 2010 /$ No\%2016\%20-\%202010.aspx

34. Report on the monitoring and testing of ruminants for the presence of transmissible spongiform encephalopathy (TSE) in the EU in 2008. Brussels: Health and Consumer Protection Directorate-General. Available from: http://ec.europa.eu/food/ food/biosafety/tse_bse/docs/annual_report_tse2008_en.pdf

35. Brandel J-P, Heath CA, Head MW, Levavasseur E, Knight R, Laplanche J-L, et al. Variant Creutzfeldt-Jakob disease in France and the United Kingdom: Evidence for the same agent strain. Ann. Neurol. 2009;65(3):249-56.

36. Lillquist PP, Thomas N, Belay ED, Schonberger LB, Morse D. Barriers to autopsy: Creutzfeldt-Jakob disease in New York state. Neuroepidemiology. 2006;26(4):207-11. 